\title{
Theory of Young Clusters
}

\author{
Matthew R. Bate \\ School of Physics, University of Exeter, Exeter EX4 4QL, United \\ Kingdom
}

Ian A. Bonnell

School of Physics and Astronomy, University of St Andrews, North Haugh, St Andrews, Fife, KY16 9SS, United Kingdom

\begin{abstract}
We review recent results from hydrodynamical calculations of the formation of young stellar clusters. The calculations present a highly dynamical picture of star formation where the mass function of stars originates from competitive accretion between protostars and dynamical ejections which halt accretion. Large star clusters form hierarchically; a molecular cloud forms many small clusters that later merge into one large cluster, erasing the initial substructure. Using calculations that resolve fragmentation down to the opacity limit, we examine the implications of dynamical star formation for the IMF, the formation of brown dwarfs and close binaries, and the sizes of protoplanetary discs. Finally, we discuss the dependence of the results on the initial conditions.
\end{abstract}

\section{Introduction}

Hydrodynamical calculations of star cluster formation are very computationally intensive and have only recently become possible. Chapman et al. (1992) performed the first hydrodynamical calculations to follow the formation of large numbers of protostars. However, they could not follow the evolution until these objects had reached their final states. Bonnell et al. (1997, 2001a,b, 2002) ignored the initial fragmentation process and performed calculations that began with protoclusters consisting of 10-1000 protostars deeply embedded in molecular gas. They were interested in how the masses of the protostars evolved as they accreted gas. They found that even starting with equal-mass objects, competitive accretion between stars quickly produced a distribution of masses similar to the stellar initial mass function (IMF). Furthermore, the calculations produced young clusters that were mass-segregated with the most massive stars in the centre of the cluster, as is frequently observed. Klessen et al. (1998, 2000, 2001), performed calculations that began with clumpy and turbulent molecular clouds, each forming $\sim 100$ objects. They were able to follow their evolution until most of the gas had been used up in the star formation process. They found that the IMF of their objects originated from a combination of fragmentation, competitive accretion, and dynamical interactions that would eject objects from the dense gas. The resulting IMFs were roughly log-normal, reminiscent of the 
stellar IMF, with a mean mass of order the mean Jeans mass of the cloud. However, these calculations did not have the resolution to follow small-scale fragmentation, most binaries, or circumstellar discs.

In this proceedings, we review results from the most recent hydrodynamical calculations of cluster formation. After a brief discussion of the method used to perform the calculations, we discuss the results from a simulation that follows the fragmentation of a large-scale turbulent molecular cloud to form more than 400 stars, the largest number to date. This calculation illustrates that large clusters may form by merging of smaller subclusters, however, it is unable to resolve brown dwarfs or most binaries and circumstellar discs. We then turn to calculations that follow the formation of somewhat smaller clusters $(\sim 50$ stars), but resolve beyond the opacity limit for fragmentation (Low \& LyndenBell 1976). The opacity limit occurs when molecular gas ceases to collapse isothermally and begins to heat up. The dynamical collapse is stopped and a pressure-supported fragment forms with an initial mass of $\approx 0.005 \mathrm{M}_{\odot}$ and radius of $\approx 5 \mathrm{AU}$ (Larson 1969). The fragment cannot collapse further to form a star (or brown dwarf) until its central temperature exceeds that required for molecular hydrogen to dissociate. This allows a 'second collapse' to form the star. Fragmentation during this second phase of collapse is thought to be inhibited by the high thermal energy content of the gas and angular momentum transport via gravitational torques (Boss 1988; Bate 1998; Bate, in preparation). Thus, the opacity limit results in a minimum mass for the initial mass function of $\sim 5 \mathrm{MJ}_{\mathrm{J}}$ and resolving the calculation down to the opacity limit means that we should capture the formation of all stars and brown dwarfs. Furthermore, because the initial radius of the fragments is $\approx 5 \mathrm{AU}$, fragmentation should not be able to form binaries with initial separations $\lesssim 10 \mathrm{AU}$.

\section{Calculations}

\subsection{The code and initial conditions}

The calculations were performed using a parallelised version of the smoothed particle hydrodynamics (SPH) code described in Bate, Bonnell, \& Price (1995) on the United Kingdom Astrophysical Fluids Facility (UKAFF). High-density bound objects (the pressure-supported fragments that become stars and brown dwarfs) consisting of many SPH gas particles are replaced by 'sink' particles. These interact with the rest of the calculation only via gravity and accrete any SPH gas particles that come within their accretion radii.

The initial conditions consist of uniform-density, spherical molecular clouds at temperatures of $10 \mathrm{~K}$. Most clouds contain $50 \mathrm{M}_{\odot}$ of gas; the cloud that forms more than 400 stars contains $1000 \mathrm{M}_{\odot}$. We impose supersonic divergence-free random Gaussian velocity fields with a power spectrum $P(k) \propto k^{-4}$. In threedimensions, the velocity dispersion varies with distance, $\lambda$, as $\sigma(\lambda) \propto \lambda^{1 / 2}$, in agreement with the observed Larson scaling relations for molecular clouds.

\subsection{Resolution}

The calculation that formed more than 400 stars used 500,000 particles to model the $1000 \mathrm{M}_{\odot}$ cloud. Thus; it could not resolve objects with masses less than 

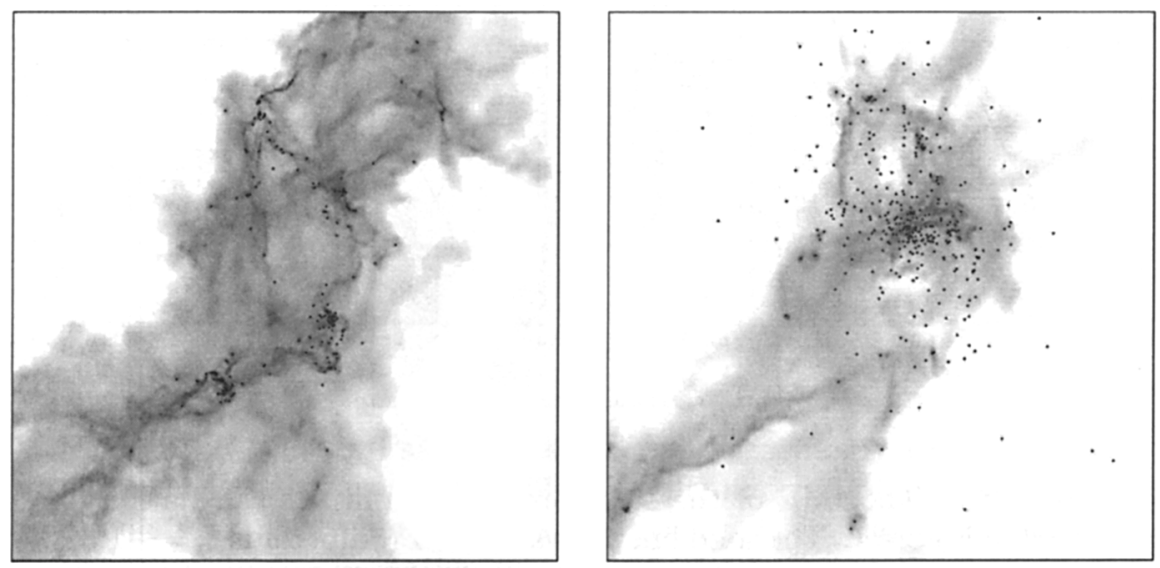

Figure 1. Snapshots of the collapse and fragmentation of a $1000 \mathrm{M}_{\odot}$ cloud to form a star cluster containing over 400 stars (Bonnell, Bate \& Vine 2003). The turbulence produces hierarchical structure in the gas; dense cores collapse and fragment to form small dense star clusters; these clusters merge, erasing the substructure and producing one large star cluster.

$\approx 0.1 \mathrm{M}_{\odot}$ or fragmentation that occurred on length scales less than $\sim 100 \mathrm{AU}$. The equation of state remained isothermal throughout the calculation.

In all the other calculations, to mimic the opacity limit for fragmentation, the equation of state changed from isothermal to barotropic with a polytropic index of $\eta=7 / 5$ at $\rho=10^{-13} \mathrm{~g} \mathrm{~cm}^{-3}$ (Bate, Bonnell \& Bromm 2003). Once the density in a pressure-supported fragment passed $\rho_{\mathrm{s}}=10^{-11} \mathrm{~g} \mathrm{~cm}^{-3}$, we replaced the fragment with a 'sink' particle. We could not follow a fragment's collapse to the actual formation of a star (as done in Bate 1998) while simultaneously following the evolution of the large-scale cloud: the range of dynamical timescales would be too large. We also resolved the local Jeans mass throughout these calculations (Bate \& Burkert 1997). The minimum Jeans mass occurs at the maximum density during the isothermal phase of the collapse, $\rho=10^{-13}$ $\mathrm{g} \mathrm{cm}^{-3}$, and is $0.0011 \mathrm{M}_{\odot}$ (1.1 Jupiter masses). The Jeans mass must be resolved by a minimum of $\approx 75 \mathrm{SPH}$ particles (Bate, Bonnell, \& Bromm 2003). Thus, we used $3.5 \times 10^{6}$ particles to model the $50 \mathrm{M}_{\odot}$ clouds.

\section{The formation of large star clusters}

Bonnell, Bate \& Vine (2003) followed the collapse and fragmentation of a 1000 $\mathrm{M}_{\odot}$ turbulent cloud to form a cluster of more than 400 stars. The turbulence results in the formation of hierarchical structure within the cloud. The high-density regions of this structure (dense molecular cores) are gravitationally bound and collapse to form small clusters of stars (Figure 1a). Within these clusters, the protostars interact via competitive accretion and some members are ejected via dynamical interactions as seen in smaller-scale calculations (Bate et al. 2003). At the same time as these interactions are occuring within each 


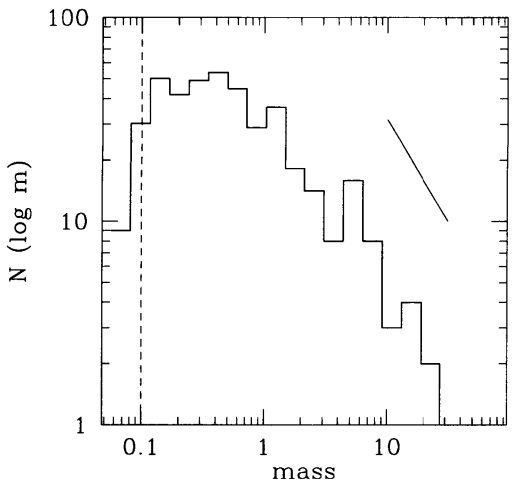

Figure 2. The IMF of the stars produced from the calculation depicted in Figure 1. The solid line shows $\Gamma=-1$ (Salpeter is $\Gamma=-1.35$ ). Objects with masses $<0.1 \mathrm{M}_{\odot}$ are unresolved in this calculation.

cluster, the velocity dispersion between the stellar clusters is small, and they fall towards each other and merge into one large cluster (Figure 1b). After 2.5 free-fall times of the initial cloud, most of the substructure has been erased and all that is left is a single cluster consisting of $\approx 400$ stars.

Such hierarchical fragmentation may be the way in which all large clusters form, e.g., the Orion Trapezium Cluster. Even though there is no sign of substructure in the Trapezium Cluster now (Bate, Clarke \& McCaughrean 1998), this simulation and the N-body simulations of Scally \& Clarke (2002) show that such initial substructure is erased very rapidly.

If large star clusters do form via merging of smaller clusters, it has important implications for interactions between forming stars. The stellar densities in the subclusters before they merge into one large cluster can be up to two orders of magnitude higher than the mean stellar density in the cluster as a whole. This dramatically increases the probability of close encounters between young stars. Bonnell et al. (2003) found that nearly $1 / 2$ of the stars had an encounter closer than $200 \mathrm{AU}$; these would truncate circumstellar discs to $\lesssim 60 \mathrm{AU}$ in radius. A high frequency of close encounters is also seen in the smaller, but fully-resolved, calculations of smaller clusters (see Section 4.4). For stars with masses $>3 \mathrm{M}_{\odot}$, the encounters tended to be even closer; more than $90 \%$ had encounters closer than $200 \mathrm{AU}$.

\section{The first fully-resolved cluster formation calculation}

Bate, Bonnell \& Bromm (2002a,b; 2003) performed the first fully-resolved calculation of star cluster formation. It followed the fragmentation of a $50 \mathrm{M}_{\odot}$ cloud to form 50 stars and brown dwarfs. The calculation was fully-resolved in the sense that fragmentation was followed down to the opacity limit for fragmentation (masses of a few Jupiter masses). Thus, all stars and brown dwarfs that should have formed in the cloud were modelled. The calculation also modelled binaries as close as $1 \mathrm{AU}$, and resolved circumstellar discs with radii $\gtrsim 20 \mathrm{AU}$. Animations of the calculation can be downloaded from http://www.astro.ex.ac.uk/people/mbate 

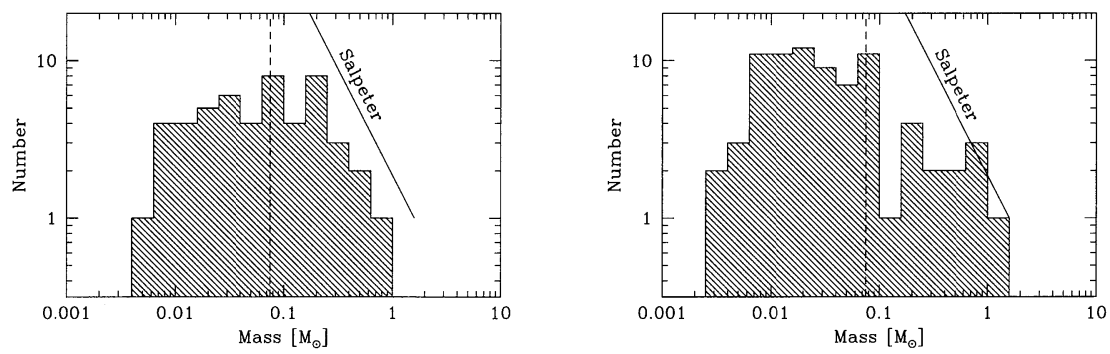

Figure 3. The IMFs of star clusters whose progenitor clouds had mean thermal Je ans masses of $1 \mathrm{M}_{\odot}$ (left) and $1 / 3 \mathrm{M}_{\odot}$ (right). These calculations resolve beyond the opacity limit for fragmentation and thus capture the formation of all stars and brown dwarfs. Reducing the mean thermal Jeans mass increases the fraction of brown dwarfs.

\subsection{The initial mass function}

In the left panel of Figure 3, we plot the IMF obtained from the calculation. It is consistent with $\mathrm{d} N / \operatorname{d} \log M=M^{\Gamma}$ where $\Gamma=-1.35$ for $M>0.5 \mathrm{M}_{\odot}, \Gamma=0.0$ for $0.006<M<0.5 \mathrm{M}_{\odot}$, and there are no objects below the opacity limit for fragmentation $\left(\approx 0.005 \mathrm{M}_{\odot}\right)$. The Salpeter slope is $\Gamma=-1.35$. This mass function is consistent with recent determinations of the IMF in young stellar clusters and star-forming regions (e.g. Luhman et al. 2000). Furthermore, there are roughly equal numbers of stars and brown dwarfs, a result also supported by observations (Reid et al. 1999).

\subsection{The formation mechanism and properties of brown dwarfs}

Although the mean Jeans mass in the cloud was $1 \mathrm{M}_{\odot}$, the calculation produced many brown dwarfs. The formation mechanism of the brown dwarfs was discussed in detail by Bate et al. (2002a). They found that the brown dwarfs formed in dense gas where the local Jeans mass was lower than that in the cloud as a whole. Roughly three quarters formed via the fragmentation of massive gravitationally-unstable circumstellar discs, while the remainder formed in dense filaments of molecular gas. However, in either case, the objects had to avoid accreting to stellar masses. This was accomplished by the objects forming in, or quickly falling into, unstable multiple systems where they were dynamically ejected from the cloud before they had been able to accrete to stellar masses. This formation mechanism was discussed by Reipurth \& Clarke (2001), although they could only speculate on its efficiency and the ways in which the multiple systems might form. Bate et al. (2002a) show that this mechanism is capable of producing the observed frequency of brown dwarfs.

The close dynamical interactions that occur during the ejection process have two important implications for the properties of brown dwarfs. First, binary brown dwarf systems should be rare, $\sim 5 \%$. Current observations suggest a frequency of $15 \pm 7 \%$ (Close et al. 2003). With only $\approx 20$ brown dwarfs formed in the hydrodynamical calculation and around twice this number surveyed observationally, both the theoretical and observational fractions are currently limited 
by small number statistics. Second, the frequency of young brown dwarfs with large circumstellar discs (greater than $\approx 20 \mathrm{AU}$ in radius) should also be low at $\sim 5 \%$. There are two main reasons for this. First, to avoid becoming stars the brown dwarfs must be ejected from the cloud soon after their formation and, thus, many do not have time to accrete the high angular momentum gas required to form large discs. Second, the majority of the dynamical encounters that eject the brown dwarfs occur at separations $<20$ AU so that any existing large disc is truncated.

\subsection{The formation mechanism and properties of close binaries}

As discussed in Section 1, the opacity limit for fragmentation sets a minimum initial binary separation of $\approx 10 \mathrm{AU}$. However, Bate et al. (2002b) found that their calculation produced 7 close binary systems (separations $<10 \mathrm{AU}$ ). Rather than forming directly by fragmentation, these systems formed from initially wider multiple systems through a combination of accretion (reducing the separation of the system), the interaction of binaries and triples with circumbinary and circumtriple discs (which also reduces the separation of the embedded system), and dynamical interactions (which can harden binaries). Together, these mechanisms can lead to efficient decay of wide multiple systems to form close systems. The frequency of close binaries at the end of the calculation was $7 / 43 \approx 16 \%$. This is in agreement with the observed frequency of close $(<10 \mathrm{AU})$ binaries of $\approx 20 \%$ (Duquennoy \& Mayor 1991), demonstrating that close binaries need not be created by fragmentation in situ.

These formation mechanisms lead to several consequences for the properties of close binaries (Bate et al. 2002b). First, there is a preference for mass ratios near unity due to the mass-equalising effect of accretion (Bate 2000) and dynamical exchange interactions which favour the ejection of the lowest mass object. This gives a natural explanation for the observation that close binaries (periods $\lesssim 10$ years) tend to have higher mass ratios than wider binaries (Mazeh et al. 1992). Second, the close binary frequency increases with primary mass due to successive dynamical exchanges. Finally, many of the close binaries are members of multiple systems. Although the true frequency of wide companions to close binaries is not yet well known, many close binaries do have wider components (e.g. Mayor \& Mazeh 1987; Tokovinin 2000).

\subsection{The sizes of protoplanetary discs}

The calculation resolved circumstellar discs with radii $\gtrsim 20 \mathrm{AU}$. Although many of the stars and brown dwarfs formed with large discs initially, dynamical interactions truncated many of the discs (Bate et al. 2003). At the end of the calculation, of the stars that had been ejected from the cloud (and thus have reached their final states) only 2 out of 10 systems (20\%) had resolved discs. For the brown dwarfs, the fraction was even lower with only 1 out of $18(\approx 5 \%)$. Even if objects that were still evolving when the calculation was stopped are included, these fractions only increase to $\approx 40 \%$ for stars and $\approx 17 \%$ for brown dwarfs. Thus, the majority of the stars and brown dwarfs, in this relatively dense star-forming region $\left(\approx 10^{3}\right.$ stars $\left./ \mathrm{pc}^{-3}\right)$, do not retain discs large enough to form our solar system. Stellar densities of this order are found in the dense cores of the $\rho$ Ophiuchus star-forming region. Furthermore, if the Trapezium 
Cluster was assembled from a group of sub-clusters, as discussed in Section 3, close dynamical interactions should have also truncated the discs of its stars and brown dwarfs. The Trapezium Cluster is the only star-forming region where we currently have information on the size distribution of discs. We know that at least $80 \%$ of its stars have discs due to their infrared excesses (Lada et al. 2000), however, of $\approx 300$ stars the HST only resolves discs around $\approx 40$ stars. HST can resolve discs down to radii of about $40 \mathrm{AU}$ in Orion, thus, the implication is that although most stars in the Trapezium Cluster have discs, only $\sim 10 \%$ are larger than $40 \mathrm{AU}$ in radius (Rodmann \& McCaughrean, in preparation). This is consistent with the above numerical results and has serious implications for the number of stars with planetary systems like our own.

\section{Variation with initial conditions}

The calculation of Bate et al. (2002a,b; 2003) began with a reasonable estimate for the conditions in molecular clouds and obtained stellar properties that are in reasonable agreement with observations. However, the question arises as to how the stellar properties depend on the conditions in the molecular cloud. Varying the mean thermal Jeans mass, the gas opacity, and/or the properties of the turbulence may lead to differences in stellar properties. Perhaps the most fundamental question about star formation is what determines the characteristic or mean 'stellar' mass? Does it depend on the opacity limit for fragmentation (and, hence, the metallicity of the gas) or on the properties of the molecular clouds (e.g. their mean thermal Jeans mass and/or the properties of the turbulence)?

Recently, Bate \& Bonnell, in preparation, performed a calculation identical to that of Bate et al. (2002a,b; 2003), but with a smaller cloud so that the mean thermal Jeans mass was reduced by a factor of 3 . The IMFs obtained from the original calculation and the new calculation are compared in Figure 3. It can be seen that the calculation with the lower thermal Jeans mass produces a larger fraction of brown dwarfs. A Kolmogorov-Smirnov test shows that this result is significant at the $98.1 \%$ level (i.e. $2.4 \sigma$ ). However, despite a reduction in the mean thermal Jeans mass by a factor of 3 , the mean object mass is only $15 \%$ lower in the second calculation. Thus, although the mean 'stellar' mass does seem to depend on the mean thermal Jeans mass in the progenitor cloud, it is only a very weak dependence. This dependence on the mean Jeans mass is consistent with Briceno et al. (2002) which finds there are a factor of two fewer brown dwarfs in Taurus compared to the Orion Trapezium Cluster, the latter of which has a higher mass density and hence a smaller inferred Jeans mass.

A third calculation is underway to investigate the dependence on the opacity limit for fragmentation. The gas heats at a density 9 times lower than in the original calculation, raising the minimum fragment mass by a factor of 3 to $\approx 15$ $\mathrm{M}_{\mathrm{J}}$. Preliminary results show the mean 'stellar' mass increases, although the calculation has not run far enough to determine accurately the factor.

A fourth calculation will be begun in October 2003 to investigate the dependence of the stellar properties on the properties of the turbulence in the molecular cloud. Together, these calculations should dramatically increase our knowledge of the origin of the properties of stars and brown dwarfs and how the star formation process occurs. 


\section{References}

Bate, M. R. 1998, ApJ, 508, L95

Bate, M. R. 2000, MNRAS, 314, 33

Bate, M. R., \& Burkert, A. 1997, MNRAS, 508, L95

Bate, M. R., Bonnell, I. A., \& Bromm, V. 2002a, MNRAS, 332, L65

Bate, M. R., Bonnell, I. A., \& Bromm, V. 2002b, MNRAS, 336, 705

Bate, M. R., Bonnell, I. A., \& Bromm, V. 2003, MNRAS, 339, 577

Bate, M. R., Bonnell, I. A., \& Price, N. M. 1995, MNRAS, 277, 362

Bate, M. R., Clarke, C. J., \& McCaughrean, M. J. 1998, MNRAS, 297, 1163

Bonnell, I. A., \& Bate, M. R. 2002, MNRAS, 336, 659

Bonnell, I. A., Bate, M. R., \& Vine, S. G. 2003, MNRAS, 343, 413

Bonnell, I. A., Bate, M. R., Clarke, C. J., \& Pringle, J. E. 1997, MNRAS, 285, 201

Bonnell, I. A., Bate, M. R., Clarke, C. J., \& Pringle, J. E. 2001a, MNRAS, 323, 785

Bonnell, I. A., Clarke, C. J., Bate, M. R., \& Pringle, J. E. 2001b, MNRAS, 324, 573

Boss, A. P. 1988, ApJ, 331, 370

Briceno, C., Luhman, K. L., Hartmann, L., Stauffer, J. R., Kirkpatrick, J. D. 2002, ApJ, 580, 317

Chapman, S., Pongracic, H., Disney, M., Nelson, A., Turner, J., \& Whitworth, A. 1992, Nature, 359, 207

Close, L. M., Siegler, N., Freed, M., \& Biller, B. 2003, ApJ, 587, 407

Duquennoy, A., \& Mayor M. 1991, A\&A, 248, 485

Klessen, R. S., \& Burkert A. 2000, ApJS, 128, 287

Klessen, R. S., \& Burkert A. 2001, ApJ, 549, 386

Klessen, R. S., Burkert, A., \& Bate, M. R. 1998, ApJ, 501, L205

Lada C. J., Muench A. A., Haisch K. E., Lada E. A., Alves J., Tollestrup E. V., Willner S. P. 2000, AJ, 120, 3162

Larson, R. B. 1969, MNRAS, 145, 271

Low, C., \& Lynden-Bell, D. 1976, MNRAS, 176, 367

Luhman, K. L., Rieke, G. H., Young, E. T., Cotera, A. S., Chen, H., Rieke, M. J., Schneider, G., \& Thompson, R. I. 2000, ApJ, 540, 1016

Mayor, M., \& Mazeh, T. 1987, A\&A, 171, 157

Mazeh, T., Goldberg, D., Duquennoy, A., \& Mayor, M. 1992, ApJ, 401, 265

Reid, I. N., Kirkpatrick, J. D., Liebert, J., Burrows, A., Gizis, J. E., Burgasser, A., Dahn, C. C., Monet, D., Cutri, R., Beichman, C. A., \& Skrutskie, M. 1999, ApJ, 521, 613

Reipurth, B., \& Clarke, C. 2001, AJ, 122, 432

Scally, A., \& Clarke, C. 2002, MNRAS, 334, 156

Tokovinin, A. A. 2000, A\&A, 360, 997 